\title{
Foveal Avascular Zone in Normal Tension Glaucoma Measured by Optical Coherence Tomography Angiography
}

\author{
Maja Zivkovic, ${ }^{1,2}$ Volkan Dayanir, ${ }^{3}$ Tolga Kocaturk, ${ }^{4}$ Marko Zlatanovic, ${ }^{2}$ \\ Gordana Zlatanovic, ${ }^{1,2}$ Vesna Jaksic, ${ }^{5}$ Marija Radenkovic, ${ }^{2}$ Predrag Jovanovic, ${ }^{1,2}$ \\ Sanja Sefic Kasumovic, ${ }^{6}$ Mladjan Golubovic, ${ }^{7}$ and Svetlana Jovanovic ${ }^{8}$
}

\author{
${ }^{1}$ Medical Faculty, Department of Ophthalmology, University of Nis, Nis, Serbia \\ ${ }^{2}$ Ophthalmology Clinic, Clinical Center Nis, Nis, Serbia \\ ${ }^{3}$ BATIGOZ Health Group Eye Clinic, Izmir, Turkey \\ ${ }^{4}$ Department of Ophthalmology, Adnan Menderes University Medical School, Aydin, Turkey \\ ${ }^{5}$ Medical Faculty, Department of Ophthalmology, University of Belgrade, Belgrade, Serbia \\ ${ }^{6}$ Eye Clinic "Dr. Sefic", Sarajevo, Bosnia and Herzegovina \\ ${ }^{7}$ Center for Anesthesiology and Reanimatology, Clinical Center Nis, Nis, Serbia \\ ${ }^{8}$ Department of Ophthalmology, Faculty of Medical Sciences, University of Kragujevac, Kragujevac, Serbia
}

Correspondence should be addressed to Svetlana Jovanovic; drsvetlanajovanovic@yahoo.com

Received 6 September 2017; Revised 22 November 2017; Accepted 27 November 2017; Published 17 December 2017

Academic Editor: Mitsuru Nakazawa

Copyright (c) 2017 Maja Zivkovic et al. This is an open access article distributed under the Creative Commons Attribution License, which permits unrestricted use, distribution, and reproduction in any medium, provided the original work is properly cited.

Aim. To measure diameter of foveal avascular zone (FAZ), FAZ area, and vessel density using Optical Coherence Tomography Angiography (OCT-A) in patients with normal tension glaucoma (NTG) and to establish the possible role of OCT-A in diagnosis and follow-up of patients with NTG. Methods. Twenty-one eyes of 21 patients with NTG and 30 eyes of 30 healthy subjects underwent complete ophthalmic examination as well as OCT-A on ZEISS AngioPlex. $3 \times 3$ macula scans were used to measure vertical, horizontal, and maximum diameter of FAZ by two graders. Mean values and interobserver variability were analyzed. Image J was used for analysis of FAZ area and vessel density. Results. Mean vertical diameter $(t=5.58, p<0.001)$, horizontal diameter $(t=3.59, p<0.001)$, maximum diameter $(t=5.94, p<0.001)$, and FAZ area $(t=5.76, p<0.001)$ were statistically significantly enlarged in the NTG group compared to those in the control group. Vessel density $(t=-5.80, p<0.001)$ was statistically significantly decreased in the NTG group compared to that in the control group. Conclusion. OCT-A could have an important role in the future in diagnosis of patients with NTG. In patients with NTG, there is larger FAZ area, while the vessel density is reduced in comparison to the control group.

\section{Introduction}

Normal tension glaucoma (NTG), also called low-tension glaucoma, is a chronic, progressive optic neuropathy with optic nerve deterioration and glaucomatous visual field defects [1]. Values of intraocular pressure (IOP) do not exceed the normal range of $21 \mathrm{mmHg}$ [2]. Vascular dysfunction has been proposed as one of the most important factors in the development and progression of NTG3. This makes the study of blood flow in NTG extremely essential. The mechanisms underlying the abnormal ocular blood flow [3-5] in NTG are still not clear, but the risk factors for glaucomatous optic neuropathy likely include oxidative stress [6], vasospasm [7], and endothelial dysfunction [8].

According to the vascular theory, damage results from low or fluctuating ocular blood flow (OBF) causing ischemia and reperfusion injury in optic nerve head $(\mathrm{ONH})$, respectively [9]. The role of vasospasm and ischemia as the primary cause of such a vascular failure in the systemic micro- and macrocirculation [10] in NTG leads to chronic oxidative stress affecting $\mathrm{ONH}$ and ganglion cells. 
Several techniques (color Doppler imaging (CDI), scanning laser ophthalmoscopy, fundus fluorescein angiography (FFA), and laser Doppler flowmetry) have been used to measure retrobulbar and intraocular hemodynamics in patients with glaucoma [11]. All the methods currently in use to measure ocular blood flow (OBF) have inherent limitations and measure different aspects of OBF [11].

In the last year, ophthalmology had a revolutionary discovery, Optical Coherence Tomography Angiography (OCTA). We can measure dimensions of the foveal avascular zone (FAZ) with great reproducibility for the first time [12]. Since vascular theory is one of the proposed theories in pathogenesis of NTG, OCT-A might help us to better understand the pathogenesis of NTG. Until now, few papers have studied peripapillary vessel density in primary open angle glaucoma (POAG), but no paper has studied dimensions of FAZ in patients with NTG $[12,13]$. To the best of our knowledge, this is the first study evaluating FAZ in NTG patients using OCTA.

\section{Aim of the Paper}

The aim of this paper is to measure diameter size of FAZ, FAZ area, and vessel density using OCT-A in patients with NTG and to establish possible role of OCT-A in diagnosis of patients with NTG.

\section{Methods}

This is a cross-sectional case control study. Study was conducted in a group of patients with confirmed NTG and in subjects without glaucoma that were age and gender matched. Twenty-one eyes of 21 patients with NTG and 30 eyes of 30 healthy subjects underwent complete ophthalmic examination as well as OCT-A on Zeiss AngioPlex, CIRRUS HDOCT Model 5000 instrument (Carl Zeiss Meditec, Dublin, CA). Considering that FAZ can vary in size and shape, control group was established with a bigger number of healthy persons. Ocular examinations were performed in the period from June 2016 to December 2016 at the Ophthalmology Eye Hospital-Clinic Maja, Nis, Serbia. This study was approved by the Ethics Committee from Ophthalmology Eye HospitalClinic Maja, Nis, Serbia, following the tenets of the Declaration of Helsinki with informed consent obtained from all the participants. All the patients were with previously diagnosed NTG and complete cardiological, neurological, and rheumatological examination already done, as well as color Doppler imaging. All patients were treated with topical antiglaucoma drug $\alpha 2$-agonist brimonidine $0.2 \%$ for at least one year and a maximum of two years, without any other topical or systemic medication taken in the last year.

Each patient underwent a complete ophthalmic examination including best corrected visual acuity (BCVA), IOP measurement by Goldman applanation tonometry, gonioscopy, visual field testing using Swedish interactive thresholding algorithm (SITA-standard) central 24-2 Humphrey perimetry (Humphrey field analyzer II, Carl Zeiss Meditec, Dublin, CA, USA), biomicroscopy, fundus examination with a plus 90-diopter lens, multicolor disc photograph, and OCT-A on
Zeiss AngioPlex, CIRRUS HD-OCT Model 5000 instrument (Carl Zeiss Meditec, Dublin, CA). Diagnostic criteria for NTG follow the criteria of the European Glaucoma Society [1]: (1) patients older than 35 years, (2) normal IOP without treatment, less than $21 \mathrm{mmHg}$, (3) optic nerve head damage typical of glaucoma, (4) visual field defects typical of glaucoma, (5) gonioscopy, open anterior chamber angle, and (6) no history of steroid use. Patients with systemic disease with ocular involvement like diabetes and neurological diseases capable of causing visual field loss or optic disc deterioration and other eye disease (except glaucoma), opacification of ocular media, intraocular surgery, refractive errors more than $+/-2 \mathrm{D}$, and history of ocular trauma were excluded from the study. The data was stratified based on patient Caucasian race.

Inclusion criteria for the control group (CG) were (1) no history or evidence of retinal and eye pathology, (2) no history of intraocular surgery, (3) IOP $\leq 21 \mathrm{mmHg}$, (4) having to be free of optic disc damage and without any systemic and neurological diseases capable of causing visual field loss or optic disc deterioration, (5) intact neuroretinal rim and normal retinal nerve fiber layer (RNFL) thickness, and (6) normal standard automated perimetry (defined as a glaucoma hemifield test within normal limits and a pattern standard deviation within 95\% confidence-interval limits).

OCT angiography was scanned by a $68 \mathrm{kHz}$ Cirrus HDOCT 5000-based Optical Micro Angiography (OMAG) prototype system. Automated segmentation of the superficial and deep capillary plexus was used for analysis. Images obtained were checked for quality (Signal Strength more than 6/10), as well as absence of artifacts.

Currently, OCT-A Zeiss AngioPlex allows access to $3 \times$ $3,6 \times 6$, and $8 \times 8$ scans. In this study, considering that it was very important to get accurate measurement of FAZ, $3 \times 3$ scans were exclusively used as they give the best view of the FAZ. We have analyzed the dimensions of FAZ manually by measuring vertical and horizontal diameters and used Image J for analysis of FAZ area and vessel density. Also considering the phenomenon of capillary drop out in patients with NTG, we introduced the concept of maximum diameter that would fit the largest diameter of FAZ for a given FAZ analyzed (Figures 1(a)-1(c)). Two experienced ophthalmologists who were blind to the diagnoses made all the measurements of FAZ diameters (vertical, horizontal, and maximum) in order to determine interobserver variability. Superficial vascular network of angiography was used since it has been proven that there is a high interobserver variability in measuring of the deep vascular network, primarily due to the shadow artifacts caused by vessels of superficial vascular network. Superficial vascular network supplies the superficial structures, namely, RNFL, ganglion cell layer, and inner plexiform layer (IPL), that are affected by glaucoma. Mean values of vertical, horizontal, and maximum diameters were used for comparison.

All $3 \times 3$ OCT-A images were exported from the system as a Joint Photographic Experts Group file into the National Institutes of Health Image J 1.50 (a publically available image processing program developed by National Institutes of Health, Bethesda, Maryland, USA) software. The FAZ area and vessel density were calculated using a previously 


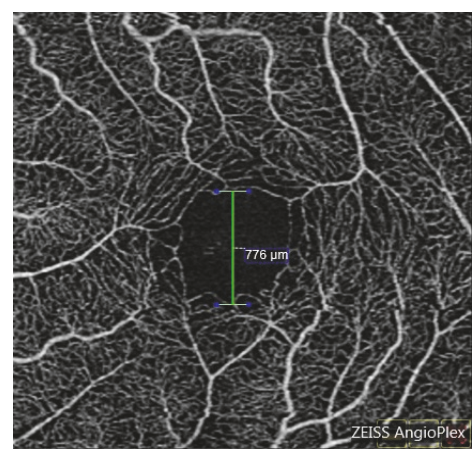

(a)

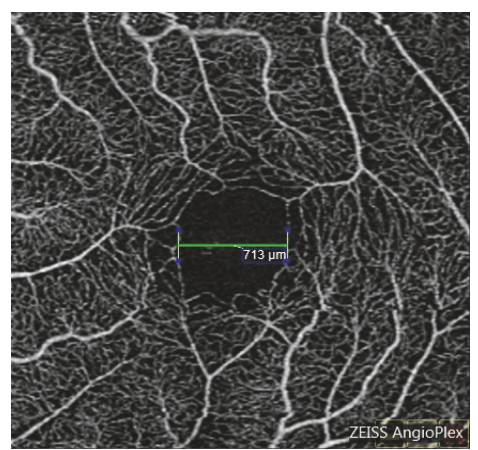

(b)

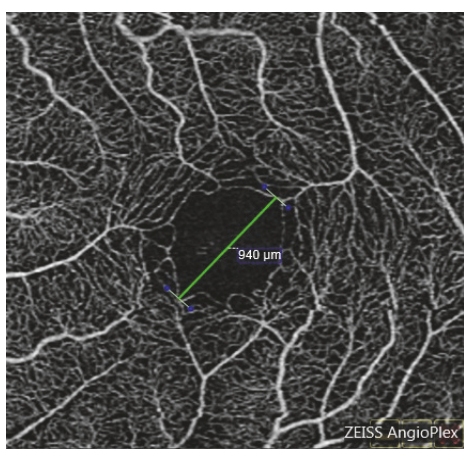

(c)

FIGURE 1: Measurement of (a) vertical, (b) horizontal, and (c) maximum diameter of foveal avascular zone.

published method [14]. FAZ area was manually outlined by the polygon selection tool. The dimension of the FAZ was expressed as square millimeters [15]. To calculate vessel density, images were binarized through a threshold strategy similarly to other studies $[14,15]$. The image was converted from 8-bit into red green blue (RGB) colour type. The adjusting threshold tool set to mean was applied; the darkbackground option was selected. The FAZ area was colored to pure blue. White pixels were considered as vessel, black pixels were considered as background, and blue pixels were automatically excluded from the analysis. Vessel density was expressed as the ratio of vessel pixels to the total area (Figures $2(a)-2(f))$.

3.1. Statistical Analysis. Statistical analysis was performed with the statistical package SPSS 22 for Windows. Primary data obtained were analyzed by descriptive statistical methods and methods for testing hypotheses. From the descriptive statistical methods, the following were used: measures of central tendency, measures of variability, and structure indicators expressed as a percentage. To determine the normality of distribution, Kolmogorov-Smirnov test was used. To test differences between 2 arithmetic means, the $t$-test for independent samples (Independent-Samples $t$-test) was used. To test differences of arithmetical mean between 2 measurements, $t$-test for dependent samples (Paired-Samples $t$ test) was used. Chi-square test of homogeneity was examined whether the two groups differ according to the proportion of the variable of interest. To test interobserver reliability, double mixed model intraclass correlation coefficient (ICC) was used.

Correlation analysis was performed by measuring the correlation between two variables. The Pearson's correlation coefficient was used to determine the direction and strength of the connection. The conclusion was done at the level of statistical significance of 0.05 .

\section{Results}

4.1. Patient Demographics and Baseline Characteristics of the Group. NTG group included 21 eyes of 11 female and 10 male patients. Mean age was $70.1 \pm 6.4$ years (mean ages of women
TABLE 1: Demographic and baseline characteristics.

\begin{tabular}{lccc}
\hline & $\begin{array}{c}\text { NTG group } \\
(n=21)\end{array}$ & $\begin{array}{c}\text { Control group } \\
(n=30)\end{array}$ & $P$ value \\
\hline Number of eyes (patients) & 21 & 30 & \\
Eye (left/right) & $11 / 10$ & $15 / 15$ & $1.00^{\mathrm{a}}$ \\
Men age (SD), years & $70.1(6.4)$ & $69.5(5.4)$ & $0.70^{\mathrm{b}}$ \\
Gender (female/male) & $11 / 10$ & $16 / 14$ & $0.61^{\mathrm{a}}$ \\
\hline
\end{tabular}

${ }^{\mathrm{a}} \chi^{2}$-test, ${ }^{\mathrm{b}} t$-test, NTG $=$ normal tension glaucoma, and $\mathrm{SD}=$ standard deviation.

and men were $69.6 \pm 6.4$ and $70.5 \pm 6.7$ years, resp.). Control group consisted of 30 eyes of 16 female and 14 male subjects. Mean age was $69.5 \pm 5.4$ years (mean ages of women and men were $71.6 \pm 5.2$ and $67.0 \pm 4.6$ years, resp.). There was no statistically significant difference between the NTG and control groups with respect to gender proportion $\left(\chi^{2}=0.46, p=\right.$ $0.61)$ and age $(t=0.40, p=0.70)$. In the NTG and control groups, there was no statistically significant difference in the participation of the left and right eyes $\left(\chi^{2}=0.00, p=1.00\right)$ (Table 1).

Statistically significant difference was not observed between the two measurements done within NTG and control groups, respectively, for vertical $(t=-1.32, p=0.238$; $t=-1.90, p=0.074)$, horizontal $(t=-1.19, p=0.124 ; t=$ $-1.92, p=0.069)$, and the maximum diameters $(t=-0.78$, $p=0.422 ; t=-1.79, p=0.086$ ) (Table 2).

Measurements done by two independent observers in the NTG and control groups, respectively, for vertical ( $r=0.99$, $p<0.001 ; r=0.99, p<0.001)$, horizontal $(r=0.77$, $p<0.001 ; r=0.99, p<0.001)$, and the maximum diameter $(r=0.99, p<0.001 ; r=0.99, p<0.001)$ were significantly positively correlated.

Mean foveal avascular zone vertical diameter $(t=5.58$, $p<0.001)$, horizontal diameter $(t=3.59, p<0.001)$, maximum diameter $(t=5.94, p<0.001)$, and foveal avascular zone area $(t=5.76, p<0.001)$ in $3 \times 3$ macula superficial scan were all statistically significantly enlarged in NTG group compared to those in the control group. Vessel density $(t=$ $-5.80, p<0.001)$ was statistically significantly decreased 


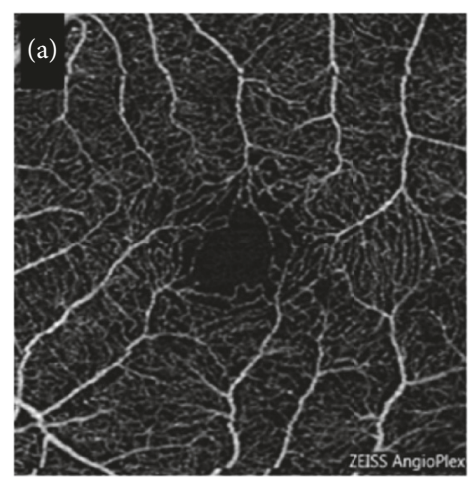

(a)

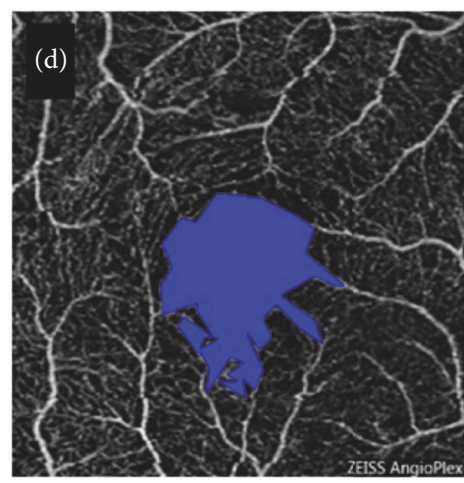

(d)

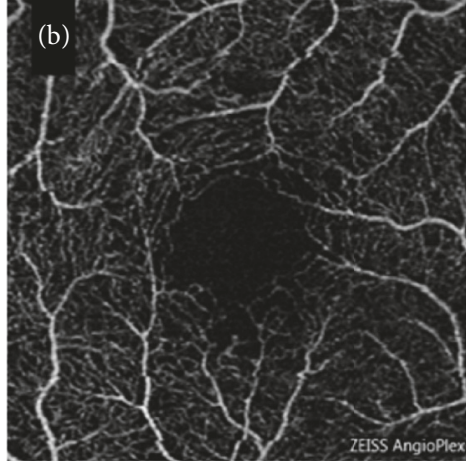

(b)

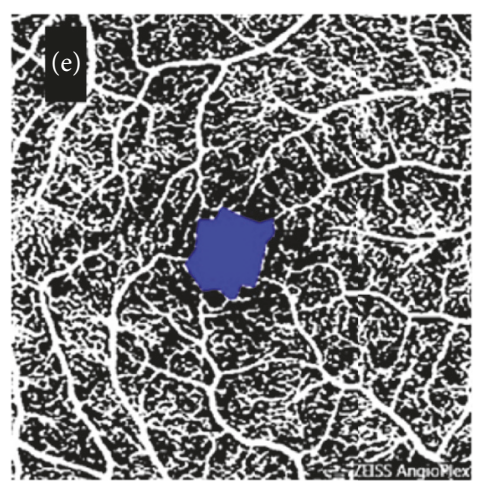

(e)

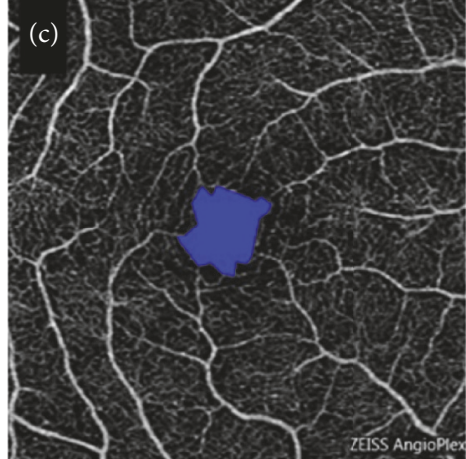

(c)

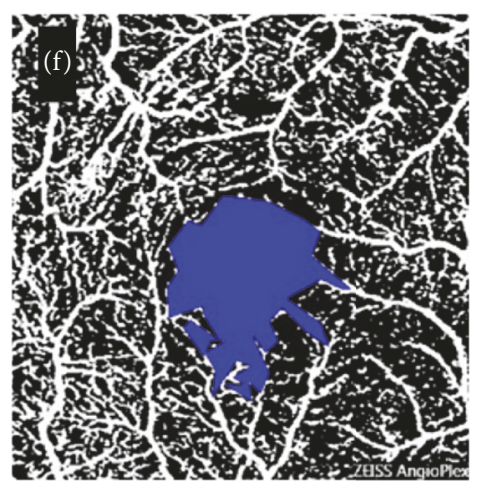

(f)

FIGURE 2: A $3 \times 3$ macular OCT-A scan of a normal eye (a) and NTG eye (b) is seen. Area of FAZ of the same normal eye (c) and NTG eye (d) as outlined by Image J program and shown in blue. Binarized image for the same normal eye (e) and NTG eye (f) showing vessels in white in order to calculate macular vessel density.

TABLE 2: Characteristics of vertical, horizontal, and maximum diameters measured by two independent observers.

\begin{tabular}{|c|c|c|c|c|}
\hline & $\begin{array}{c}\text { 1st observer } \\
x \pm \mathrm{SD}(\min -\max )\end{array}$ & $\begin{array}{c}\text { 2nd observer } \\
x \pm \mathrm{SD} \text { (min-max) }\end{array}$ & $P$ value & $r$ \\
\hline \multicolumn{5}{|l|}{ NTG group } \\
\hline Vertical diameter & $\begin{array}{c}648.98 \pm 123.76 \\
\quad(510-1000)\end{array}$ & $\begin{array}{c}650.33 \pm 118.87 \\
(525-989)\end{array}$ & $0.238^{\mathrm{a}}$ & $0.99^{\mathrm{b} *}$ \\
\hline Horizontal diameter & $\begin{array}{c}631.50 \pm 64.44 \\
(520-840)\end{array}$ & $\begin{array}{c}630.92 \pm 69.89 \\
(541-829)\end{array}$ & $0.124^{\mathrm{a}}$ & $0.77^{\mathrm{b} *}$ \\
\hline Maximum diameter & $\begin{array}{c}731.45 \pm 111.02 \\
(624-1055)\end{array}$ & $\begin{array}{c}732.23 \pm 110.13 \\
(608-1034)\end{array}$ & $0.422^{\mathrm{a}}$ & $0.99^{\mathrm{b} *}$ \\
\hline \multicolumn{5}{|l|}{ Control group } \\
\hline Vertical diameter & $\begin{array}{l}512.02 \pm 89.45 \\
(336-706)\end{array}$ & $\begin{array}{c}513.43 \pm 84.23 \\
(353-690)\end{array}$ & $0.074^{\mathrm{a}}$ & $0.99^{\mathrm{b} *}$ \\
\hline Horizontal diameter & $\begin{array}{l}546.54 \pm 86.65 \\
(427-804)\end{array}$ & $\begin{array}{l}548.68 \pm 83.35 \\
(442-799)\end{array}$ & $0.069^{\mathrm{a}}$ & $0.99^{\mathrm{b} *}$ \\
\hline Maximum diameter & $\begin{array}{c}592.11 \pm 95.32 \\
(440-802)\end{array}$ & $\begin{array}{c}582.45 \pm 90.24 \\
(470-819)\end{array}$ & $0.086^{\mathrm{a}}$ & $0.99^{\mathrm{b} *}$ \\
\hline
\end{tabular}

${ }^{\mathrm{a}} t$-test, ${ }^{\mathrm{b}} r=$ Pearson's correlation coefficient, $\mathrm{SD}=$ standard deviation, and NTG = normal tension glaucoma; ${ }^{*} p<0.001$.

in the NTG group compared to that in the control group (Table 3).

Interobserver agreement was high for all measurements (vertical diameter, horizontal diameter, and maximum diameter) (Table 4).

\section{Discussion}

Vascular factors play a significant role in the development of NTG. Blood supply of the eye is mainly from the ophthalmic artery (OA), a branch of the internal carotid artery (ICA). The 
TABLE 3: Characteristics of the average value of vertical, horizontal, and maximum diameter, foveal avascular zone area, and vessel density in NTG and control group.

\begin{tabular}{|c|c|c|c|}
\hline $\begin{array}{l}\text { Average between } 2 \\
\text { measurements }\end{array}$ & $\begin{array}{c}\text { NTG group } \\
x \pm \mathrm{SD} \\
(\min -\max )\end{array}$ & $\begin{array}{c}\text { Control group } \\
x \pm \mathrm{SD} \\
(\min -\mathrm{max})\end{array}$ & $P$ value \\
\hline Vertical diameter & $\begin{array}{c}649.43 \pm 112.55 \\
(517.5-994.5)\end{array}$ & $\begin{array}{c}512.23 \pm 85.90 \\
(344.5-697.0)\end{array}$ & $<0.001^{\mathrm{a}}$ \\
\hline Horizontal diameter & $\begin{array}{c}630.34 \pm 63.10 \\
(530.5-834.5)\end{array}$ & $\begin{array}{c}559.77 \pm 83.52 \\
(434.5-801.5)\end{array}$ & $<0.001^{\mathrm{a}}$ \\
\hline Maximum diameter & $\begin{array}{l}731.45 \pm 112.96 \\
(616.0-1044.5)\end{array}$ & $\begin{array}{c}586.83 \pm 88.39 \\
(455.5-810.5)\end{array}$ & $<0.001^{\mathrm{a}}$ \\
\hline Foveal avascular zone area & $\begin{array}{l}0.412 \pm 0.060 \\
(0.295-0.472) \\
\end{array}$ & $\begin{array}{l}0.292 \pm 0.048 \\
(0.178-0.399)\end{array}$ & $<0.001^{\mathrm{a}}$ \\
\hline Vessel density & $\begin{array}{c}39.55 \pm 3.81 \\
(35.40-41.90)\end{array}$ & $\begin{array}{c}46.21 \pm 3.36 \\
(43.10-50.80)\end{array}$ & $<0.001^{\mathrm{a}}$ \\
\hline
\end{tabular}

${ }^{\mathrm{a}} t$-test, NTG = normal tension glaucoma, $x=$ mean, and $\mathrm{SD}=$ standard deviation.

TABLE 4: Interobserver reliability.

\begin{tabular}{lcc}
\hline & $\begin{array}{c}\text { NTG group } \\
(n=21) \\
\text { ICC }(95 \% \mathrm{CI})\end{array}$ & $\begin{array}{c}\text { Control group } \\
(n=30)\end{array}$ \\
& 0.996 & ICC $(95 \% \mathrm{CI})$ \\
\hline Vertical diameter & $(0.991-0.999)$ & $(0.990-0.997)$ \\
Horizontal diameter & 0.989 & 0.994 \\
& $(0.974-0.995)$ & $(0.988-0.996)$ \\
Maximum diameter & 0.996 & 0.996 \\
& $(0.991-0.998)$ & $(0.992-0.998)$ \\
\hline
\end{tabular}

NTG $=$ normal tension glaucoma, ICC $=$ intraclass correlation coefficient, and $95 \% \mathrm{CI}=95 \%$ confidence interval.

OA gives rise to ciliary arteries, which supply the choroid, outer retina, and $\mathrm{ONH}$, and the central retinal artery (CRA) that supplies inner retina. Branches of CRA branch out on the RNFL level making up the superficial plexus within the ganglion cell layer while the deeper branches reach out to the inner nuclear layer and provide deep plexus $[8,10,11]$.

For retrobulbar circulation monitoring, currently the most widely used method is CDI. CDI is a perfect tool to assess the large ophthalmic vessels, such as the OA, CRA, and the short posterior ciliary arteries (SPCAs) [16]. The mean velocity and the resistivity index (RI) are the two important parameters that can be followed, except that RI does not accurately correlate with the resistance offered by the ophthalmic vessels $[17,18]$. Kocaturk et al. reported increased vascular resistance in OA in pseudoexfoliation glaucoma [19]. Many studies demonstrated that the blood flow resistance was increased and blood flow velocities were decreased in the OA and SPCAs of patients with POAG, pseudoexfoliation syndrome, and NTG $[9,17-19]$. Reduced blood flow velocities and increased RI in retrobulbar vessels in patients with NTG may explain the reason for the $\mathrm{ONH}$ fluorescein filling defects and capillary loss of the ONH of these patients [18].

Since it is proven that there are changes at the level of OA, CRA, and SPCAs, the main arteries that supply the eye with blood, great importance is given in finding adequate methods that will examine the most credible changes in intraocular blood vessels. Numerous studies have followed the retinal arteriovenous passage (AVP) times and the perfusion of retinal and choroidal microvascular beds with fluorescein angiography and found that the AVP was prolonged in NTG patients [20]. Arend et al. examined whether a macular capillary density reduction and/or peripapillary diameter changes account for the pathological retinal circulation in patients with NTG [21]. They speculated that pathological alterations of arterial or venous diameter or macular vasculature might occur in NTG, causing gliosis-like repair mechanisms, or that retinal capillary density is reduced as a sign of ischaemic disease. Capillary dropout or vasoconstriction of the retinal vasculature could contribute to increased resistance. In the study, they used digital scanning laser fluorescein angiography (Rodenstock Instruments, Ottobrunn, Germany) and the angiograms were recorded on NTSC Sony videotapes. Their results showed that the AVP time of NTG patients was significantly prolonged when compared with healthy subject data, but morphological data of FAZ and perifoveal intercapillary areas did not differ from healthy subjects; neither did peripapillary arterial and venous diameter measurements [21].

In recent years, the measurement of ocular blood flow has become easier and noninvasive owing to the development of OCT-A. OCT-A enables us to measure the dimension of FAZ, capillary-free area in central macula with high accuracy. Up to now, there was not any information in the literature on FAZ area and macular vessel density at NTG measuring with OCT-A, although there are many papers related to the peripapillary and macular vessel density in healthy persons $[22,23]$ and in patients with the POAG. Yarmohammadi et al. have found that reduced peripapillary and macular vessel density were detectable in the perimetrically intact hemiretinae of glaucoma eyes with a single-hemifield defect [24]. Their conclusion was that OCT-A potentially shows promise for identifying focal glaucomatous damage before VF defects are detectable.

Numerous studies using OCT-A in patients with open angle glaucoma have demonstrated reduced $\mathrm{ONH}$ and peripapillary vessel densities in patients with glaucoma [25-27]. Also many studies have demonstrated that peripapillary 
angioflow density shows strong correlation with the RNFL thickness [26-28].

There are only few papers that were done with NTG patients, but the authors followed only peripapillary vessel density, not macular vessel density. Shin et al. demonstrated that peripapillary vessel density maps of superficial and deep retinal layers were significantly reduced at the 7 and 11 oclock positions in glaucomatous eyes [29]. Scripsema et al. compared perfused peripapillary capillary densities in POAG, NTG, and normal patients using OCT-A and showed that POAG and NTG patients had a reduction of perfused capillaries that progressed in size when comparing early, moderate, and severe glaucoma groups [30]. Bojikian et al. investigated optic disc perfusion differences in healthy subjects, POAG, and NTG and showed that optic disc perfusion was significantly reduced in POAG and NTG groups compared to normal controls, but no difference was seen between POAG and NTG groups with similar levels of VF damage [20]. Igarashi et al. published that flow density and the disappearance angle of the radial peripapillary capillaries were significantly and independently correlated with glaucoma-related functional and morphological changes in the optic nerve in POAG and NTG [31].

Our study is the first one that follows macular vessel density and FAZ area in NTG. In our study, larger FAZ areas with reduced vessel density were found in NTG patients. It would be possible to define an exact value of FAZ area to support the diagnosis of NTG or other types of glaucoma with larger number of patients. These vascular parameters are gradually becoming important in terms of diagnosis, progression, and treatment options in the management of the patients with glaucoma.

As far as we know, this is the first study that will shed light on further investigation about FAZ in patients with NTG. Further investigations related to the FAZ and peripapillary and macular vessel density are needed with larger numbers of patients in order to determine the role of OCT-A in glaucoma.

\section{Conclusion}

OCT-A could have an important role in the future in diagnosis of patients with NTG as well as in understanding the pathophysiology of NTG. We have shown that, for the first time in the literature, NTG patients have larger FAZ area and reduced macular vessel density compared to the control group.

\section{Disclosure}

There is no financial or proprietary interest in any materials or methods described herein.

\section{Conflicts of Interest}

The authors have no proprietary or commercial interests in any concept or product discussed in this paper.

\section{References}

[1] European Glaucoma Society, Terminology and Guidelines for Glaucoma, Editrice Dogma, Savon, Italy, 4nd edition, 2014.

[2] M. B. Shields and G. L. Spaeth, "The glaucomatous process and the evolving definition of glaucoma," Journal of Glaucoma, vol. 21, no. 3, pp. 141-143, 2012.

[3] C. D. Phelps and J. J. Corbett, "Migraine and low-tension glaucoma. A case-control study," Investigative Ophthalmology \& Visual Science, vol. 26, no. 8, pp. 1105-1108, 1985.

[4] J. Flammer, K. Konieczka, and A. J. Flammer, "The primary vascular dysregulation syndrome: implications for eye diseases," EPMA Journal, vol. 4, no. 1, article 14, 2013.

[5] M. Mozaffarieh and J. Flammer, "Ocular blood flow and glaucomatous optic neuropathy," Ocular Blood Flow and Glaucomatous Optic Neuropathy, pp. 1-105, 2009.

[6] M. Mozaffarieh and J. Flammer, "New insights in the pathogenesis and treatment of normal tension glaucoma," Current Opinion in Pharmacology, vol. 13, no. 1, pp. 43-49, 2013.

[7] Y. Delaney, T. E. Walshe, and C. O'Brien, "Vasospasm in glaucoma: clinical and laboratory aspects," Optometry and Vision Science, vol. 83, no. 7, pp. 406-414, 2006.

[8] N. Fan, P. Wang, L. Tang, and X. Liu, "Ocular blood flow and normal tension glaucoma," BioMed Research International, vol. 2015, Article ID 308505, 2015.

[9] J. C. Tsai, "Influencing ocular blood flow in glaucoma patients: The cardiovascular system and healthy lifestyle choices," Canadian Journal of Ophthalmology, vol. 43, no. 3, pp. 347-350, 2008.

[10] J. Bossuyt, G. Vandekerckhove, T. L. M. De Backer et al., "Vascular dysregulation in normal-tension glaucoma is not affected by structure and function of the microcirculation or macrocirculation at rest: A case-control study," Medicine (United States), vol. 94, no. 2, p. e425, 2015.

[11] S. Xu, S. Huang, Z. Lin, W. Liu, and Y. Zhong, "Color doppler imaging analysis of ocular blood flow velocities in normal tension glaucoma patients: a meta-analysis," Journal of Ophthalmology, vol. 2015, pp. 1-24, 2015.

[12] R. Mastropasqua, L. Toto, P. A. Mattei et al., "Reproducibility and repeatability of foveal avascular zone area measurements using swept-source optical coherence tomography angiography in healthy subjects," European Journal of Ophthalmology, vol. 27, no. 3, pp. 336-341, 2017.

[13] W. A. Samara, E. A. T. Say, C. T. L. Khoo et al., "Correlation of foveal avascular zone size with foveal morphology in normal eyes using optical coherence tomography angiography," Retina, vol. 35, no. 11, pp. 2188-2195, 2015.

[14] M. B. Parodi, M. V. Cicinelli, A. Rabiolo et al., "Vessel density analysis in patients with retinitis pigmentosa by means of optical coherence tomography angiography," British Journal of Ophthalmology, vol. 101, no. 4, pp. 428-432, 2017.

[15] M. Battaglia Parodi, A. Rabiolo, M. V. Cicinelli, P. Iacono, F. Romano, and F. Bandello, "Quantitative analysis of optical coherence tomography angiography in adult-onset foveomacular vitelliform dystrophy," Retina, 2017.

[16] F. Jimenez-Aragon, E. Garcia-Martin, R. Larrosa-Lopez, J. M. Artigas-Martín, P. Seral-Moral, and L. E. Pablo, "Role of color Doppler imaging in early diagnosis and prediction of progression in glaucoma," BioMed Research International, vol. 2013, Article ID 871689, 11 pages, 2013.

[17] I. Marjanović, M. Marjanović, A. Martinez, V. Marković, M. Božić, and V. Stojanov, "Relationship between blood pressure 
and retrobulbar blood flow in dipper and nondipper primary open-angle glaucoma patients," European Journal of Ophthalmology, vol. 26, no. 6, pp. 588-593, 2016.

[18] M. T. Nicolela, S. N. Ferrier, C. A. Morrison et al., "Effects of cold-induced vasospasm in glaucoma: the role of endothelin-1," Investigative Ophthalmology \& Visual Science, vol. 44, no. 6, pp. 2565-2572, 2003.

[19] T. Kocaturk, I. Isikligil, B. Uz, V. Dayanir, and Y. O. Dayanir, "Ophthalmic artery blood flow parameters in pseudoexfoliation glaucoma," European Journal of Ophthalmology, vol. 26, no. 2, pp. 124-127, 2015.

[20] K. D. Bojikian, C. Chen, J. C. Wen et al., "Optic disc perfusion in primary open angle and normal tension glaucoma eyes using optical coherence tomography-based microangiography," PLoS ONE, vol. 11, no. 5, p. e0154691, 2016.

[21] O. Arend, A. Remky, N. Plange, B. J Martin, and A. Harris, "Capillary density and retinal diameter measurements and their impact on altered retinal circulation in glaucoma: A digital fluorescein angiographic study," British Journal of Ophthalmology, vol. 86, no. 4, pp. 429-433, 2002.

[22] A. Shahlaee, M. Pefkianaki, J. Hsu, and A. C. Ho, "Measurement of foveal avascular zone dimensions and its reliability in healthy eyes using optical coherence tomography angiography," American Journal of Ophthalmology, vol. 161, pp. 50-55.e1, 2016.

[23] G. N. Magrath, E. A. T. Say, K. Sioufi, S. Ferenczy, W. A. Samara, and C. L. Shields, "Variability in foveal avascular zone and capillary density using optical coherence tomography angiography machines in healthy eyes," Retina, 2016.

[24] A. Yarmohammadi, L. M. Zangwill, A. Diniz-Filho et al., "Peripapillary and Macular Vessel Density in Patients with Glaucoma and Single-Hemifield Visual Field Defect," Ophthalmology, vol. 124, no. 5, pp. 709-719, 2017.

[25] X. Wang, C. Jiang, T. Ko et al., "Correlation between optic disc perfusion and glaucomatous severity in patients with openangle glaucoma: an optical coherence tomography angiography study," Graefe's Archive for Clinical and Experimental Ophthalmology, vol. 253, no. 9, pp. 1557-1564, 2015.

[26] L. Liu, Y. Jia, H. L. Takusagawa et al., "Optical coherence tomography angiography of the peripapillary retina in glaucoma," JAMA Ophthalmology, vol. 133, no. 9, pp. 1045-1052, 2015.

[27] A. Yarmohammadi, L. M. Zangwill, A. Diniz-Filho et al., "Optical coherence tomography angiography vessel density in healthy, glaucoma suspect, and glaucoma eyes," Investigative Ophthalmology \& Visual Science, vol. 57, no. 9, pp. OCT451OCT459, 2016.

[28] G. Holló, "Intrasession and between-visit variability of sector peripapillary angioflow vessel density values measured with the angiovue optical coherence tomograph in different retinal layers in ocular hypertension and glaucoma," PLoS ONE, vol. 11, no. 8, Article ID e0161631, 2016.

[29] J. W. Shin, K. R. Sung, J. Y. Lee, J. Kwon, and M. Seong, “Optical coherence tomography angiography vessel density mapping at various retinal layers in healthy and normal tension glaucoma eyes," Graefe's Archive for Clinical and Experimental Ophthalmology, vol. 255, no. 6, pp. 1193-1202, 2017.

[30] N. K. Scripsema, P. M. Garcia, R. D. Bavier et al., "Optical coherence tomography angiography analysis of perfused peripapillary capillaries in primary open-angle glaucoma and normal-tension glaucoma," Investigative Opthalmology \& Visual Science, vol. 57, no. 9, p. OCT611, 2016.

[31] R. Igarashi, S. Ochiai, Y. Sakaue et al., "Optical coherence tomography angiography of the peripapillary capillaries in primary open-angle and normal-tension glaucoma," PLOS ONE, vol. 12, no. 9, p. e0184301, 2017. 


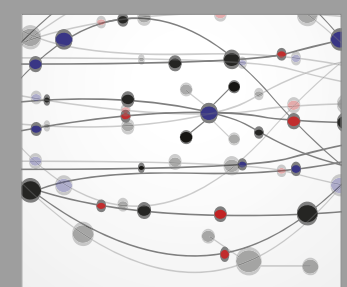

The Scientific World Journal
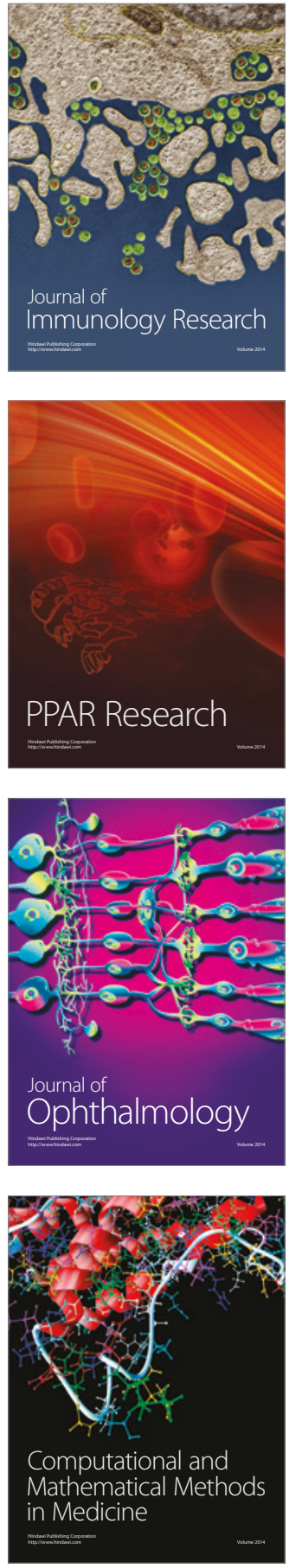

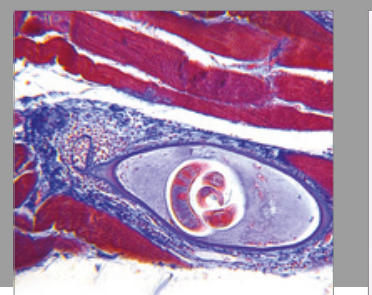

Gastroenterology Research and Practice
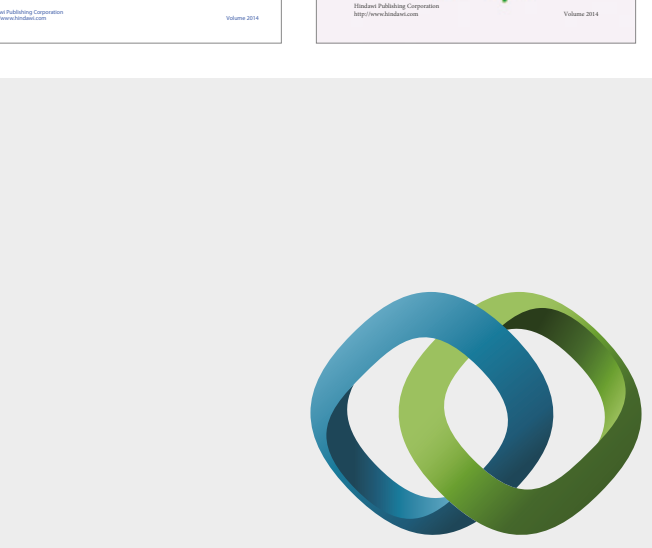

\section{Hindawi}

Submit your manuscripts at

https://www.hindawi.com
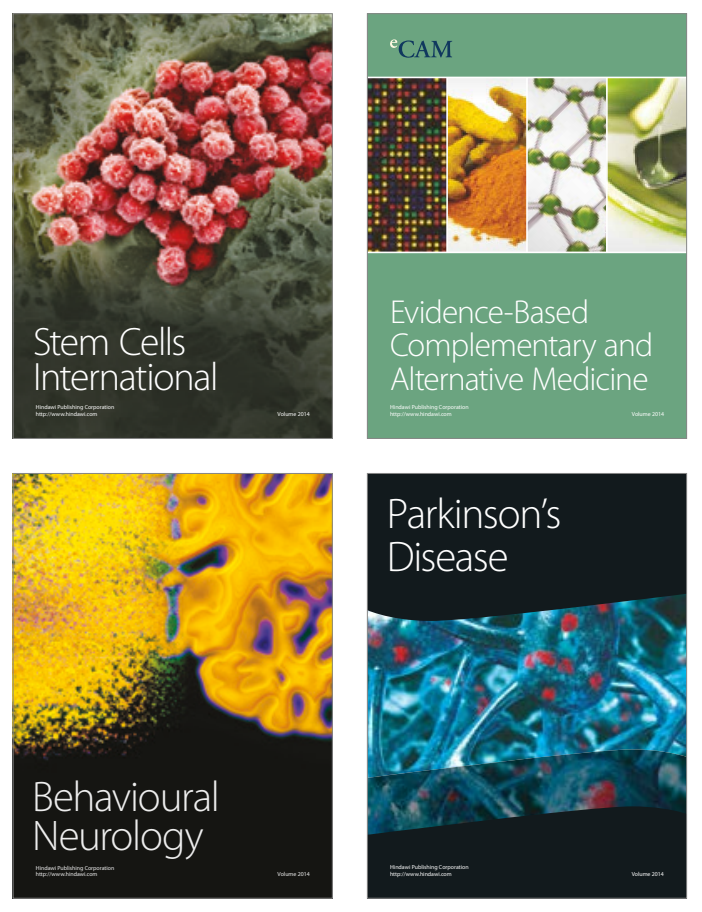
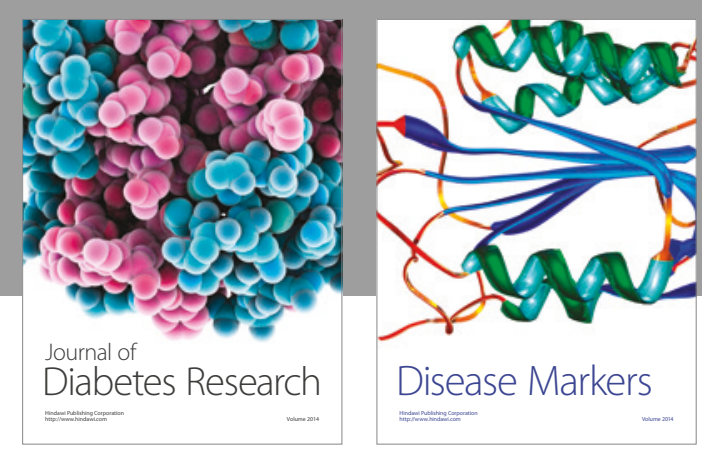

Disease Markers
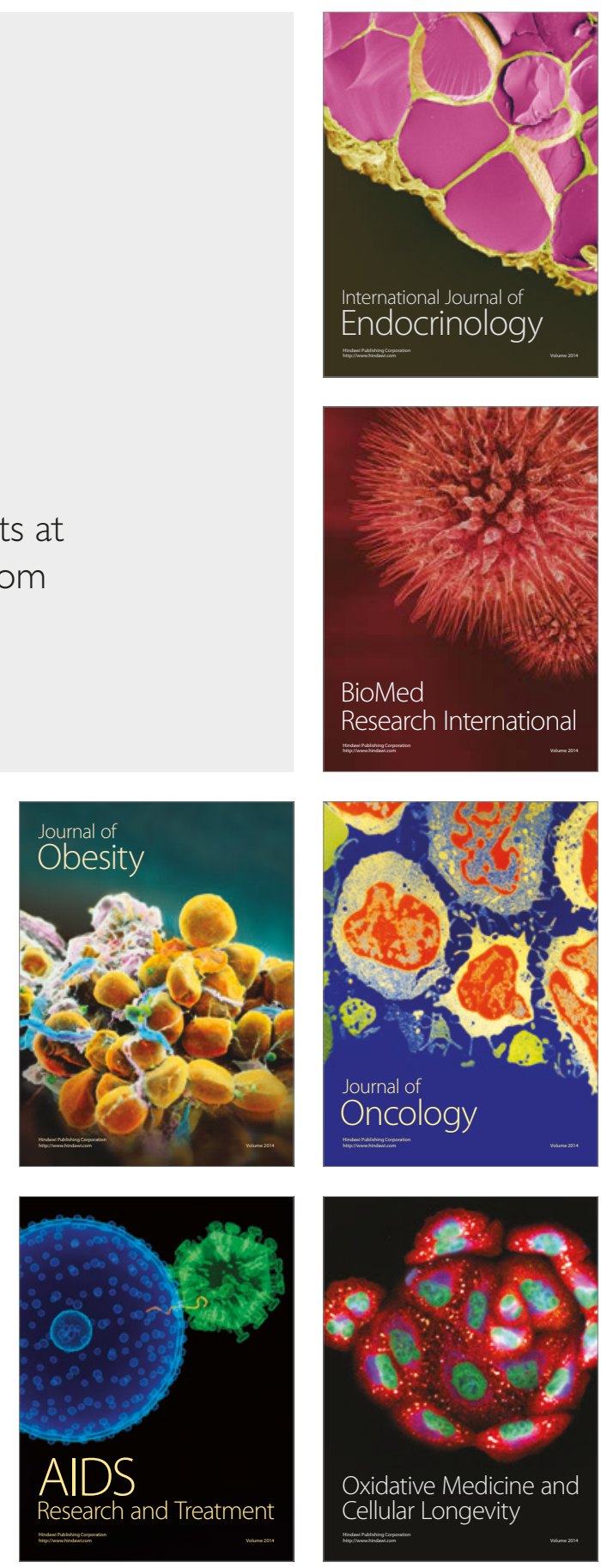